\title{
EFFECT OF ELECTROCARDIOGRAPHIC FINDINGS ON MORTALITY OF ACUTE EXACERBATION OF COPD- AN OBSERVATIONAL STUDY
}

\author{
Durga Balagopalan1, Ponneduthamkuzhy Thomas James², Anoop Muthiraparambathu
}

${ }_{1}^{1}$ Assistant Professor, Department of Pulmonology, Malabar Medical College, Modakkallur, Kozhikode, Kerala, India.

2 Professor and HOD, Department of Pulmonology, Amrita Institute of Medical Sciences, Kochi, Kozhikode, Kerala, India.

${ }^{3}$ Consultant Pulmonologist, Department of Pulmonology, MIMS Hospital, Kozhikode, Kerala, India.

\section{ABSTRACT}

\section{BACKGROUND}

An exacerbation of COPD is defined as an acute worsening of respiratory symptoms that result in additional therapy. COPD is currently the fourth leading cause of death in the world. There is a need for prospective trials in COPD based on hard clinical outcomes such as death to bring improvements in clinical managements. Chronic obstructive pulmonary disease (COPD) ranks fourth as a cause of death in the United States, behind heart disease, cancer, and stroke. COPD is a costly disease with both direct costs (value of health care resource devoted to diagnosis and management) and indirect costs (monetary consequences of disability, missed work, premature mortality and caregiver or family costs resulting from the illness 1 ). Diagnostics test for COPD is spirometry FEV1/FVC. Spirometry is costly and is not readily available in rural settings and the technique to perform spirometry involves a lot of patient's conscious effort. Whereas ECG is easily available, affordable, does not require patient's conscious effort. We wanted to predict the electrocardiographic findings predicting mortality in acute exacerbation of COPD.

\section{METHODS}

This is an observational study including 101 patients with COPD acute exacerbations admitted in our institution for a period of 1 year. Selected patients were evaluated with a detailed clinical history and a 12 lead ECG is taken. Data was collected and tabulated. Statistical analysis was done using SPSS Software Version 12.0 and Epi Info Version 3.4.1. Univariate analysis comparing various variables in survivors and non survivors was done using Chi Square test and p value and Odds ratio were calculated. Then multivariate analysis was done using linear regression analysis to find out the most important independent predictors of mortality in COPD acute exacerbations. Study was approved by institutional ethics committee.

\section{RESULTS}

In this study, all the patients were males. 34 patients died during treatment. In the study, mean age of patients was 62.33 years. Of the patients who died, 10 (29.41\%) were beedi smokers, and 24 patients were cigarette smokers. More patients belonged to the group with heavy smoking score, i.e.; $>400$. Presence of p pulmonale and RVH in ECG was found to be a predictor of poor outcome in case of a COPD acute exacerbation ( $\mathrm{p}$ value $<0.05$ ).

\section{CONCLUSIONS}

In this study, majority of the patients belonged to the age group 60-70 years. Dyspnoea was the predominant symptom of our patients. Mortality rate of COPD acute exacerbations was $33.66 \%$ in this observational study. Electrocardiographic findings of $p$ pulmonale and right ventricular hypertrophy were found to be predictors of mortality in COPD acute exacerbations by univariate analysis and multivariate analysis. In this study, electrocardiographic findings of p pulmonale and right ventricular hypertrophy were found to be important predictors of mortality in COPD acute exacerbations.

\section{KEY WORDS}

COPD Exacerbations, Mortality, ECG Findings

HOW TO CITE THIS ARTICLE: Balagopalan D, James PT, Muthiraparambathu A. Effect of electrocardiographic findings on mortality of acute exacerbation of COPD- an observational study. J. Evolution Med. Dent. Sci. 2019;8(22):1789-1793, DOI: $10.14260 /$ jemds/2019/393

\section{BACKGROUND}

An exacerbation of COPD is defined as an acute worsening of respiratory symptoms that result in additional therapy. ${ }^{1}$ Chronic obstructive pulmonary disease (COPD) ranks fourth as a cause of death in the United States, behind heart disease, cancer, and stroke.

'Financial or Other Competing Interest': None.

Submission 29-04-2019, Peer Review 20-05-2019,

Acceptance 22-05-2019, Published 03-06-2019.

Corresponding Author:

Dr. Durga Balagopalan

Assistant Professor

Department of Pulmonology, Malabar Medical College,

Modakkallur, Kozhikode, Kerala, India.

E-mail: durgabalagopalan1979@gmail.com

DOI: $10.14260 /$ jemds $/ 2019 / 393$

\section{(c) $($ ) $९$}

Additionally, since serious co-morbidities are often present in patients with COPD, many die from other diseases such as cardiac disease or cancer. Not surprisingly, multiple factors, reflective of both respiratory disease process and the substantial co-morbidity, predict survival in the disease ${ }^{5}$ With the progression of COPD comes progressive exercise limitation.

Increased respiratory rate results in ventilation-perfusion in homogeneity that causes an increase in the alveolararterial oxygen difference. Eventually, alveolar hypoxemia leads to pulmonary hypertension, which becomes manifest as cor-pulmonale. ${ }^{2}$ COPD is currently the fourth leading cause of death in the world ${ }^{2,3}$ and further increases in the prevalence and mortality of the disease can be predicted in the coming decades. ${ }^{3,4}$ Currently, men still have a higher mortality rate from COPD than women (83 vs. 57 per 100, 000), but the mortality rate is rising in women and is stable in men. ${ }^{2}$ 
Among patients with an acute exacerbation of COPD and a $\mathrm{PaCO}_{2}$ of $50 \mathrm{mmHg}$ or more, the six and 12-month mortality rates are approximately 33 and 43 percent, respectively. ${ }^{5}$ It is estimated that 14 percent of patients admitted for an exacerbation of COPD will die within three months of admission. 6,7 Survival time was independently related to severity of illness, body mass index (BMI), age, prior functional status, $\mathrm{PaO}_{2} / \mathrm{FiO}_{2}$, congestive heart failure, serum albumin, and the presence of cor-pulmonale.

COPD is a costly disease with both direct costs (Value of health care resource devoted to diagnosis and management) and indirect costs (Monetary consequences of disability, missed work, premature mortality and caregiver or family costs resulting from the illness. ${ }^{1}$ Diagnostics test for COPD is spirometry FEV1/FVC. Spirometry is costly and is not readily available in rural settings and technique to perform spirometry involves a lot of patient's conscious effort. Whereas ECG is easily available, affordable, does not require patient's conscious effort.

A complex and interrelated set of risk factors influences functional decline, exacerbation and early mortality in patients with COPD. With limited health care resources, efficient and effective management of COPD ideally involves identifying and focusing efforts on individuals who are at particular risk. ${ }^{1}$

There is a need for prospective trials in COPD based on hard clinical outcomes such as death to bring improvements in clinical managements. Better understanding of COPD is imperative. This potentially disabling and fatal disease is already epidemic in many countries and appears destined to become worldwide in coming decades given trends of smoking prevalence. ${ }^{2}$

COPD develops insidiously. However, the disease can be easily detected with simple spirometric testing before symptoms occur, and cessation of smoking can slow or even halt the disease progression and prolong survival. Once the disease is symptomatic, a coordinated, comprehensive, and individualized approach to treatment, both pharmacologic and non-pharmacologic, can increase functional status, prevent complications, and improve the quality of life. ${ }^{2}$

Exacerbations of COPD can range from those that are nuisances to those that are life threatening, but treatment can shorten the duration of illness and improve outcomes. In advanced disease, treatments including surgical approaches are directed toward relief of symptoms and prolongation of survival. Thus, although there is certainly need for improvement in our treatment of symptomatic COPD, current treatments are effective and a nihilistic attitude is not warranted. ${ }^{2}$ Mortality rate, the possible factors affecting mortality and intubation in patients with acute exacerbation of chronic obstructive pulmonary diseases (COPD) are yet unclear. So, this study intended to find out the mortality rate in patients with COPD acute exacerbations and also to find out simple clinical and laboratory parameters that may predict the mortality. After COPD becomes clinically apparent, the median survival is about 10 years. Several factors have been identified that predict poor survival in COPD. These include low FEV1, active smoking status, hypoxemia, poor nutrition, the presence of cor-pulmonale, resting tachycardia, low exercise capacity, severe dyspnoea, poor health-related quality of life, anaemia, frequent exacerbations, co-morbid illnesses, and low carbon monoxide diffusing capacity. Patients with an FEV1 less than 35 percent predicted have about 10 percent mortality per year. If a patient reports that they are unable to walk 100 meters without stopping because of breathlessness, the 5-year survival is only 30 percent.

\section{Aim of The Study}

To evaluate the electrocardiographic findings predicting mortality in acute exacerbation of COPD.

\section{METHODS}

This study was an observational study. Patients admitted in Institute of chest diseases, Medical College Calicut with signs and symptoms of chronic obstructive pulmonary disease acute exacerbation were included in the study. Study period was 1 year.

\section{Inclusion Criteria}

All cases of COPD diagnosed by clinical features and spirometry criteria as per GOLD 2006 and is on treatment for more than one year, now admitted in our institution with exacerbation were included in the study.

\section{Exclusion Criteria}

Patients with co-morbid conditions like

- Primary heart Disease.

- Cirrhosis Liver.

- Immunodeficiency.

- Renal failure.

\section{Method of Data Collection}

Patients enrolled in to the study were evaluated in detail by collecting the following-

I. A detailed history including 1.Age.

2.Sex.

3.Smoking history. 4.0ccupation.

5.Previous history of hospitalization during last 1 year. 6.Previous history of intubations.

\section{Electrocardiography}

12-lead ECG of the selected patients were taken. Patients with ECG findings of either $P$ wave amplitude $>2.5 \mathrm{~mm}$ or peaked $\mathrm{P}$ wave were considered as criteria for $\mathrm{P}$ pulmonale. $\mathrm{RVH}$ is defined by one or more of the following criteria:

- $\quad$ Right axis deviation $\geq 110^{\circ}$

- $\mathrm{R} / \mathrm{S}$ ratio in $\mathrm{V}_{1}>1$

- $\quad \mathrm{R}$ wave in $\mathrm{V}_{1} \geq 7 \mathrm{~mm}$

- $\quad S$ wave in $V_{1}<2 \mathrm{~mm}$

- $\quad$ QR pattern in $\mathrm{V}_{1}$

- $\mathrm{R}$ wave in $\mathrm{V}_{1}+\mathrm{S}$ wave in $\mathrm{V}_{5}$ or $\mathrm{V}_{6}>10.5 \mathrm{~mm}$

- $\mathrm{R} / \mathrm{S}$ ratio in $\mathrm{V}_{5}$ or $\mathrm{V}_{6} \leq 1$

- Onset of intrinsicoid deflection in $\mathrm{V}_{1}=0.035-0.055$

- $\quad$ Second rSR'in $V_{1}$ with $R^{\prime} \geq 10 \mathrm{~mm}$ 


\section{RESULTS}

In this study conducted a brief idea about the mortality rate in COPD acute exacerbation and effect of ECG findings on the mortality of COPD during its exacerbation is given.
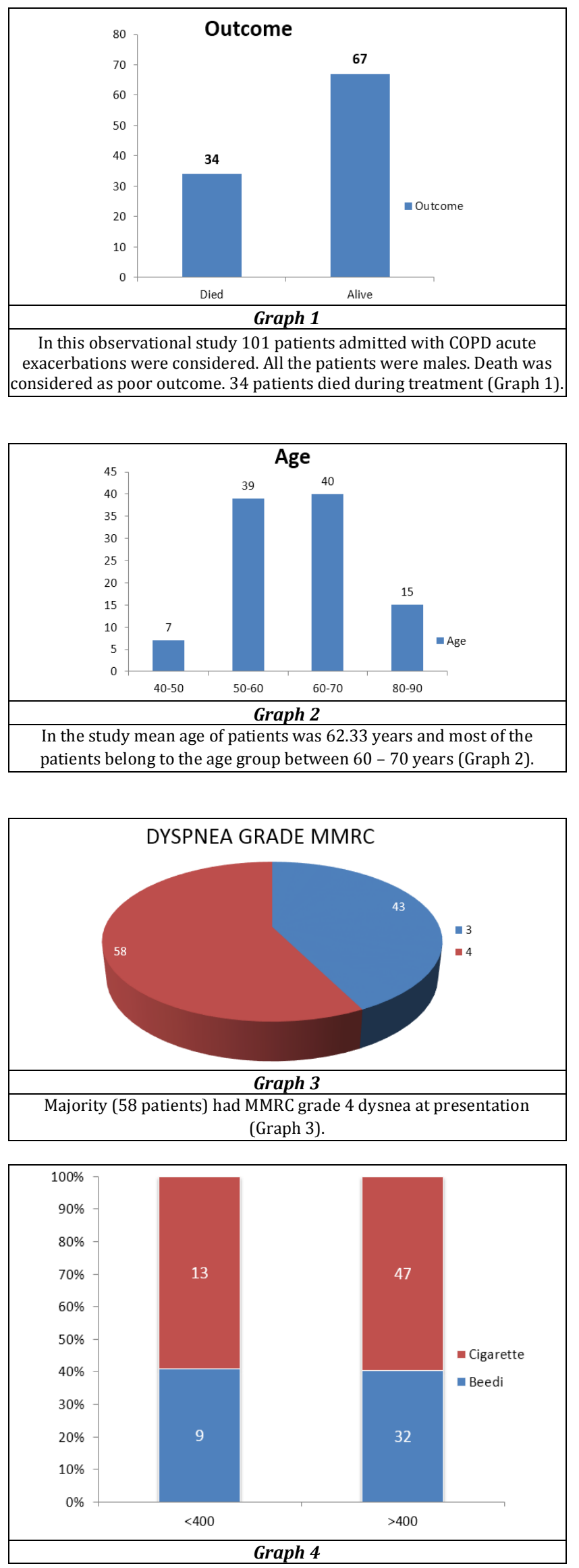
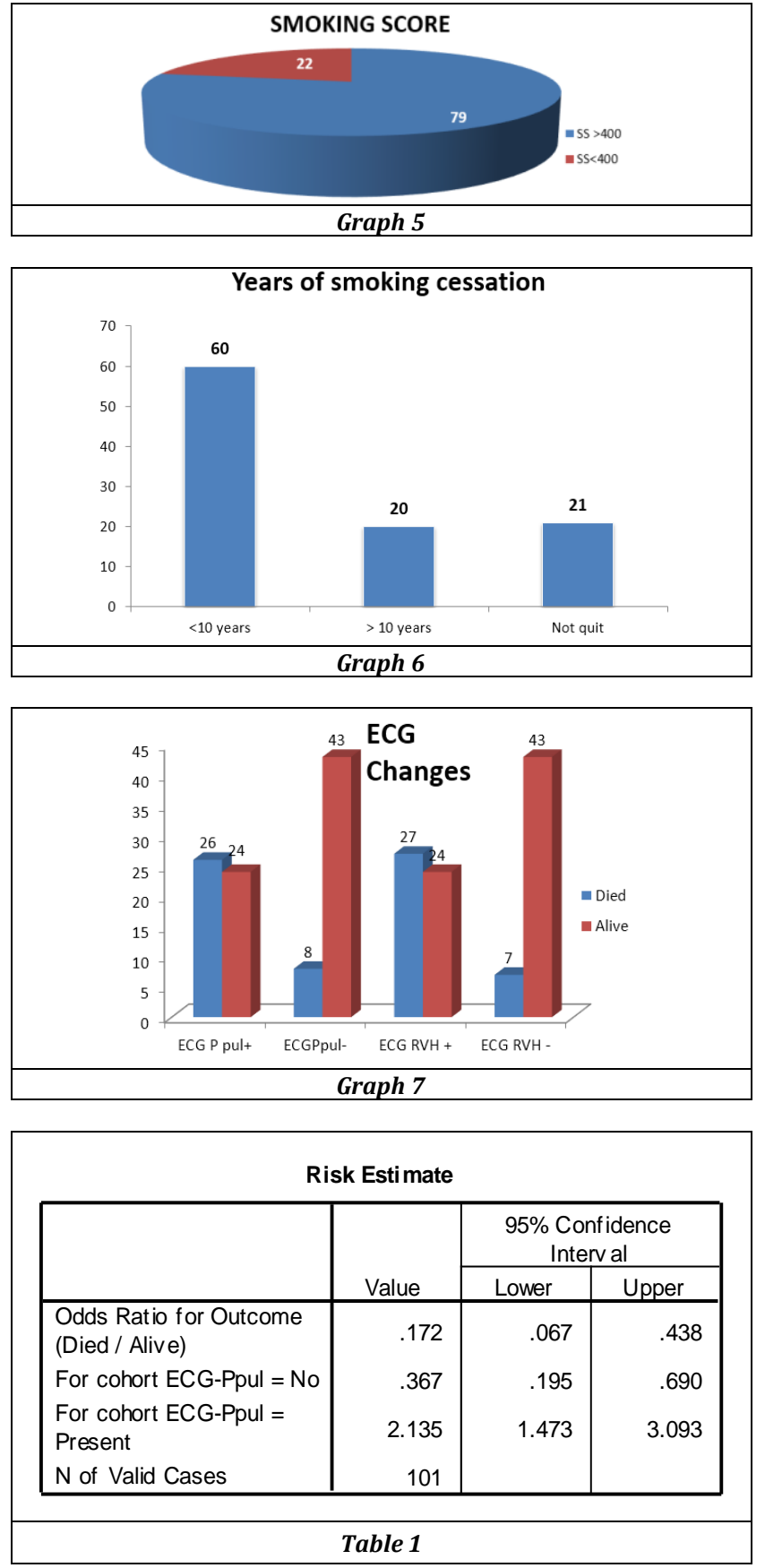

\begin{tabular}{|l|r|r|r|}
\hline \multicolumn{4}{|c|}{ Risk Estimate } \\
\hline & & \multicolumn{2}{|c|}{$\begin{array}{c}95 \% \text { Confidence } \\
\text { Interval }\end{array}$} \\
\cline { 3 - 4 } & \multicolumn{1}{|c|}{ Value } & \multicolumn{1}{|c|}{ Lower } & \multicolumn{1}{c|}{ Upper } \\
\hline Odds Ratio for Outcome & .145 & .055 & .382 \\
(Died / Alive) & .321 & .162 & .636 \\
For cohort ECG-RVH = No & .217 & 1.542 & 3.188 \\
For cohort ECG-RVH = & 2.217 \\
Present & 101 & & \\
N of Valid Cases & Table 2 \\
\hline \multicolumn{4}{|c|}{} \\
\hline
\end{tabular}

\begin{tabular}{|c|c|c|c|c|}
\hline Variable & $\begin{array}{c}\text { Correlation } \\
\text { Coefficient }\end{array}$ & Std. Error & F-Test & p-Value \\
\hline ECG Pulmonale & -0.227 & 0.078 & 8.3947 & 0.004692 \\
\hline ECG RVH & -0.227 & 0.088 & 9.53948 & 0.004652 \\
\hline Constant & 0.373 & 0.512 & 0.5296 & 0.468610 \\
\hline \multicolumn{5}{|c|}{ Table 3 } \\
\hline \multicolumn{5}{|c|}{ Correlation Coefficient: $\mathrm{r}^{\wedge} 2=0.43$} \\
\hline
\end{tabular}


By multivariate analysis also ECG changes of p pulmonale and right ventricular hypertrophy were found to be independent predictors of mortality in patients with COPD acute exacerbations ( $\mathrm{p}$ value $<0.05$ ) Table 3

ECG showed features of p pulmonale in 50 patients and features of RVH in 33 patients. 26 and 27 patients who died had features of $p$ pulmonale and RVH in their ECG respectively (Graph 7). Presence of p pulmonale and RVH in ECG was found to be a predictor of poor outcome in case of a COPD acute exacerbation ( $\mathrm{p}$ value $<0.05$ ) (Table 1 and 2 ).

$100 \%$ of patients were smokers. $59.4 \%$ were smoking cigarettes (Graph 4). Of the patients who died 10 (29.41\%) were beedi smokers and 24 patients were cigarette smokers. More number of patients belonged to the group with heavy smoking score, i.e.; $>400$ (graph 5).21 patients were still smoking at presentation (Graph 6)

\section{Statistical Analysis}

Data were collected and tabulated. Statistical analysis was done using SPSS Software version 12.0 and Epi Info version 3.4.1. Univariate analysis comparing various variables in survivors and non survivors was done using Chi Square test and $\mathrm{p}$ value and Odds ratio were calculated. Then multivariate analysis was done using linear regression analysis to find out the most important independent predictors of mortality in COPD acute exacerbations.

Study was approved by institutional ethics committee.

\section{DISCUSSION}

In this study 101 patients admitted with COPD acute exacerbations were taken into consideration. All the patients were males. Death was considered as the poor outcome. We tried to find out important predictors of mortality in patients during COPD acute exacerbations. 34 (33.66\%) patients died during treatment and 67 (66.34\%) survived the acute event. 3 patients were mechanically ventilated. All the three patients expired. UcqunI et al found out a similar in hospital mortality rate $(33.1 \%)$ for patients admitted with COPD acute hypercapnic respiratory failure in their study ${ }^{8}$. Susheel Patilet al mentioned in their study that previous studies of selected populations of patients with COPD have estimated in-hospital mortality to range from $4 \%$ to $30 \% .{ }^{9}$

\section{Demographics}

In our study mean age was 62.33 and most of the patients belonged to the age group between $60-70$ years (39.60\%). 39 (38.61\%) patients belonged to the age group of $50-60$ years. Divay Chandra et al found almost similar age group of patients in a similar study conducted by them; ie, the mean age was 61.2 years. ${ }^{10}$

$58(57.43 \%)$ patients had MMRC grade 4 dyspnoea at presentation. Rest of the 43 patients had MMRC grade 3 . Grade of dyspnoea did not predict poor outcome in these patients in our study. 41 (40.59\%) patients were smoking beedis. $60(59.41 \%)$ patients were smoking cigarettes. 79 (78.22\%) patients had a smoking score of $>400.22$ (21.78\%) patients had a smoking score $<400$. High smoking score was not associated with a poor outcome in our study. 60 (59.41\%) patients had quit smoking for only $<10$ years. $21(20.79 \%)$ patients were still smoking at the time of enrolment into the study.

\section{Electrographic Findings}

$26(76.5 \%)$ of 34 patients who died had features of corpulmonale in ECG and 27 (79.4\%) had features of right ventricular hypertrophy. Presence of these ECG findings were an important predictor of mortality in COPD exacerbations as evidenced by statistical analysis $(p=0.00)$ table 3 and 4 . Raffaele Antonelli Incalzi et al found out that the Cox regression analysis identified $\mathrm{S}_{1} \mathrm{~S}_{2} \mathrm{~S}_{3}$ pattern, right atrial overload (RAO), and alveolar-arterial oxygen gradient $\left(\mathrm{PAO}_{2}-\right.$ $\mathrm{PaO}_{2}$ ) $>48 \mathrm{mmHg}$ during oxygen therapy as the strongest predictors of death. Limitations of this study are the following: first, lack of right heart catheterization in most of our patients prevented us from assessing the relationship between ECG signs of CCP and pulmonary hypertension; second, $>2$ ECG signs of CCP coexisted in a large fraction of patients, which is expected to weaken the prognostic meaning of individual ECG signs; and third, the diagnosis of coronary artery disease based on ECG criteria might be unreliable in some CCP patients. ${ }^{11} \mathrm{~L}$. Fuso et al observed that selected co-morbid diseases and electrocardiographic signs of right ventricular hypertrophy play a major prognostic role in advanced chronic obstructive pulmonary disease. The present data were derived from patients with severe COPD and thus cannot be applied to the overall population of COPD patients. Efforts should be made to further characterize the spectrum of co-morbid diseases and to assess their prognostic role in the earlier stages of chronic obstructive pulmonary disease. The physio pathological and clinical bases of the relationship between electrocardiogram signs of right ventricular hypertrophy and death should be clarified. The prevalent type of respiratory disease, i.e. bronchitic or emphysematous, should also be identified, because it might have prognostic relevance. ${ }^{12}$ The clinical assessment of patients with chronic obstructive pulmonary diseases should include these easily measurable variables. Ram Abhishek Sharma et al concluded that if $\mathrm{P}$ pulmonale is present Chronic obstructive pulmonary disease is severe and patient may be managed accordingly even if spirometry is not available. If $\mathrm{P}$ pulmonale is present it can be concluded that COPD is severe, and patient may be managed accordingly even if spirometry is not available. If $\mathrm{P}$ pulmonale is present, patients may be started on both inhaled corticosteroids as well as on inhaled long acting $\beta 2$ agonist. In many hospitals in India ECG facilities are available but spirometry is not available, especially in rural areas which contribute a large portion of population in India. ECG does not require cooperation from patient, unlike spirometry. In India it is often difficult for patients to understand how to perform spirometry. Even after repeated attempts they are not able to do it and this can lead to wrong diagnosis. ${ }^{13}$

\section{CONCLUSION}

In this study, majority of the patients belonged to the age group 60-70 years. Dyspnoea was the predominant symptom of our patients. Mortality rate of COPD acute exacerbations was $33.66 \%$ in this observational study. Electrocardiographic findings of $\mathrm{p}$ pulmonale and right ventricular hypertrophy were found to be predictors of mortality in COPD acute exacerbations by univariate analysis and multivariate analysis. 


\section{REFERENCES}

[1] Global Strategy for diagnosis, management, and prevention of chronic obstructive pulmonary disease. 2019.

[2] Fishman AP, Elias JA, Fishman JA, et al. Fishman's Pulmonary diseases and disorders. $4^{\text {th }}$ edn. McGrawHill Professional 2008: p. 691-931.

[3] Murray CJ, Lopez AD. Evidence-based health policy-lessons from the Global Burden of Disease Study. Science 1996;274(5288):740-3.

[4] Murray CJL, Lopez AD. The global burden of disease: a comprehensive assessment mortality and disability from disease, injuries and risk factors in 1990 and projected to 2020. Cambridge, MA: Harvard University Press 1996.

[5] Connors AF Jr, Dawson NV, Thomas C, et al. Outcomes following acute exacerbation of severe chronic obstructive lung disease. The SUPPORT investigators (Study to Understand Prognoses and Preferences for Outcomes and Risks of Treatments). Am J Respir Crit Care Med 1996;154(4 Pt 1):959-67.

[6] Roberts CM, Lowe D, Bucknall CE, et al. Clinical audit indicators of outcome following admission to hospital with acute exacerbation of chronic obstructive pulmonary disease. Thorax 2002;57(2):137-41.

[7] Donaldson GC, Wedzicha JA. COPD exacerbations. 1: Epidemiology. Thorax 2006;61(2):164-8.
[8] Ucqun l, Metintas M, Moral H, et al. Predictors of hospital outcome and intubation in COPD patients admitted to respiratory ICU for acute hypercapnic respiratory failure. Respir Med 2006;100(1):66-74.

[9] Patil SP, Krishnan JA, Lechtzin N, et al. In-hospital mortality following acute exacerbations of chronic obstructive pulmonary disease. Arch Intern Med 2003;163(10):1180-6.

[10] Chandra D, Guntupalli KK, Guleria R. Hypotension is a predictor of mortality in acute exacerbations of chronic obstructive pulmonary disease. Indian J Chest Dis Allied Sci 2007;49(1):13-8.

[11] Incalzi RA, Fuso L, De Rosa $M$, et al. Electrocardiographic signs of chronic corpulmonale: a negative prognostic finding in chronic obstructive pulmonary disease. Circulation 1999;99(12):1600-5.

[12] Incalzi AR, Fuso L, De Rosa M, et al. Co-morbidity contributes to predict mortality of patients with chronic obstructive pulmonary disease. Eur Respir J 1997;10(12):2794-800.

[13] Sharma RA, Hashim Z, Sharma E, et al. Diagnosis of severity of COPD on the basis of electrocardiogram. Indian Journal of Basic \& Applied Medical Research 2013;2(6):527-30. 